Yana Antova | University of West London, UK

Anne Manyande I University of West London, UK

\title{
SEX AND ETHNIC DIFFERENCES IN GLOBAL PSYCHOLOGICAL DISTRESS BEFORE COUPLE THERAPY
}

\begin{abstract}
A couple's distress is associated with their inability to effectively communicate and successfully resolve problems. But are there any differences in levels of psychological distress between men and women of different ethnic backgrounds? This study offers a quantitative account of the effect of sex and ethnicity on the levels of psychological distress in heterosexual couples
\end{abstract}

A couple's poor communication and problem-solving skills - where both partners struggle to accept each other's differences - can lead to distress. Couple therapy has become a common undertaking nowadays as a way of resolving such distress. It is regarded as an effective approach, and a crucial locus of intervention in improving couples' mental health and well-being (Balfour \& Lanman, 2012). Typically, couple therapy aims to assist and moderate a couple's communication styles through acceptance and emotional reciprocity.

\section{Cross-national relationships under strain Normally, the main source of strain in} cross-national relationships (in addition to linguistic differences and the demand that one, or both partners use their non-native language), is the lack of common early-life socialisation into similar cultural symbols and associations, not the differences in values (which can just as easily occur in mono-ethnic relationships). Different experiences of discrimination and racism can also cause difficulties. However, the couple may not always perceive these discrepancies as a problem and may instead complain of general incompatibility or stress (Kenney \& Kenney, 2014). Therefore, acknowledging these differences is an essential requirement for a better solution in a well-balanced couple's relationship.

It is also noteworthy, that intercultural couples experience additional difficulties, such as society's perception of such relationships, as well as non-acceptance on the part of their families (Kenney \& Kenney, 2014). Nonetheless, couple therapy may not be openly welcomed by some (non-white) ethnic groups, therefore the therapist must recognise that in some instances not only the couple but the rest of the family may need to be offered therapeutic intervention (Bacigalupe \& Cámara, 2012). Since a couple's ethnic characteristics play a crucial role, a culturally competent therapist should possess specific skills, such as having good knowledge and understanding of various cultural backgrounds. In assessing clients representing minority cultural origins, specific cultural norms of the couple are important and must be examined, for example, norms for marriage, partnership roles and responsibilities (Kenney \& Kenney, 2014). 


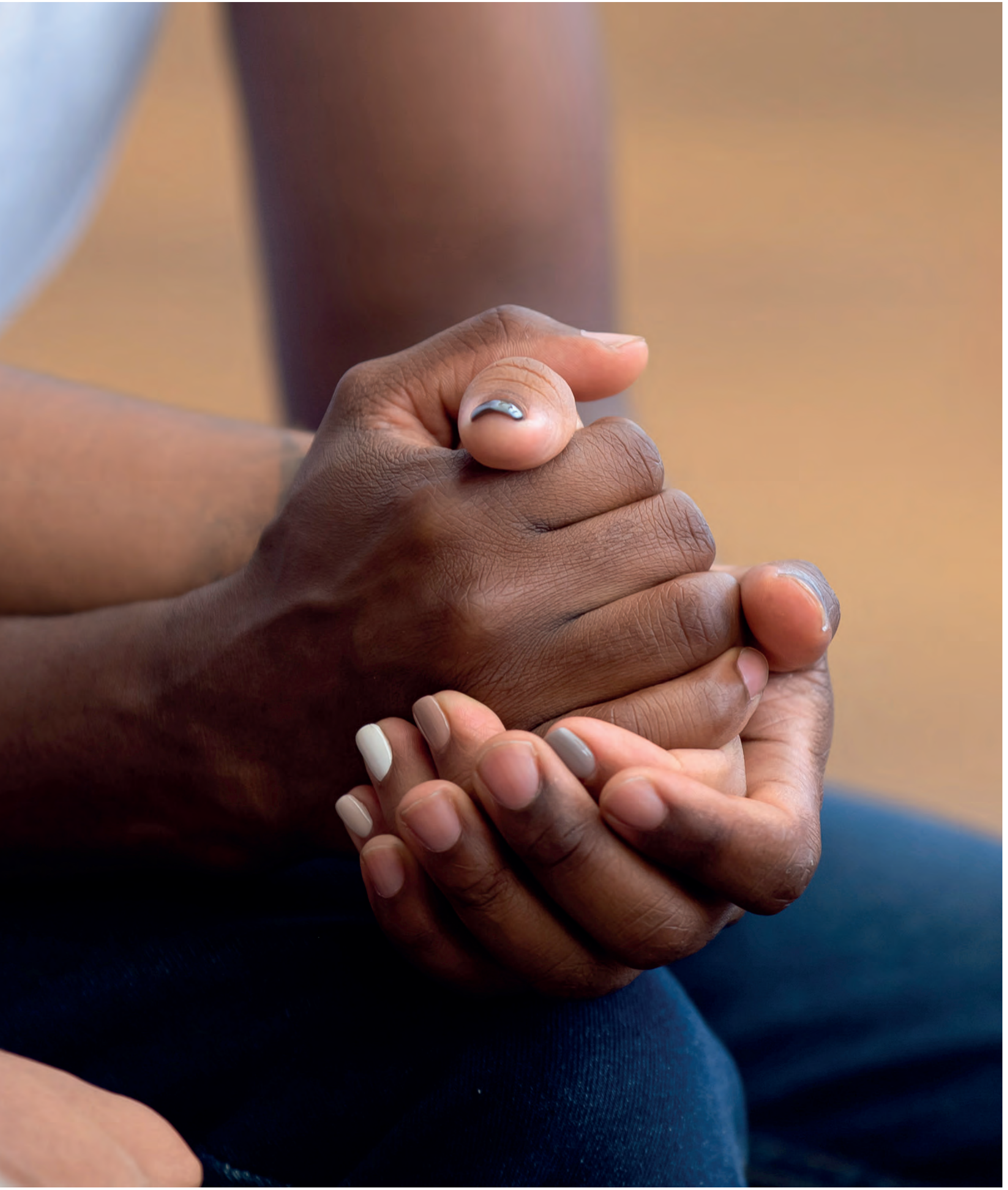




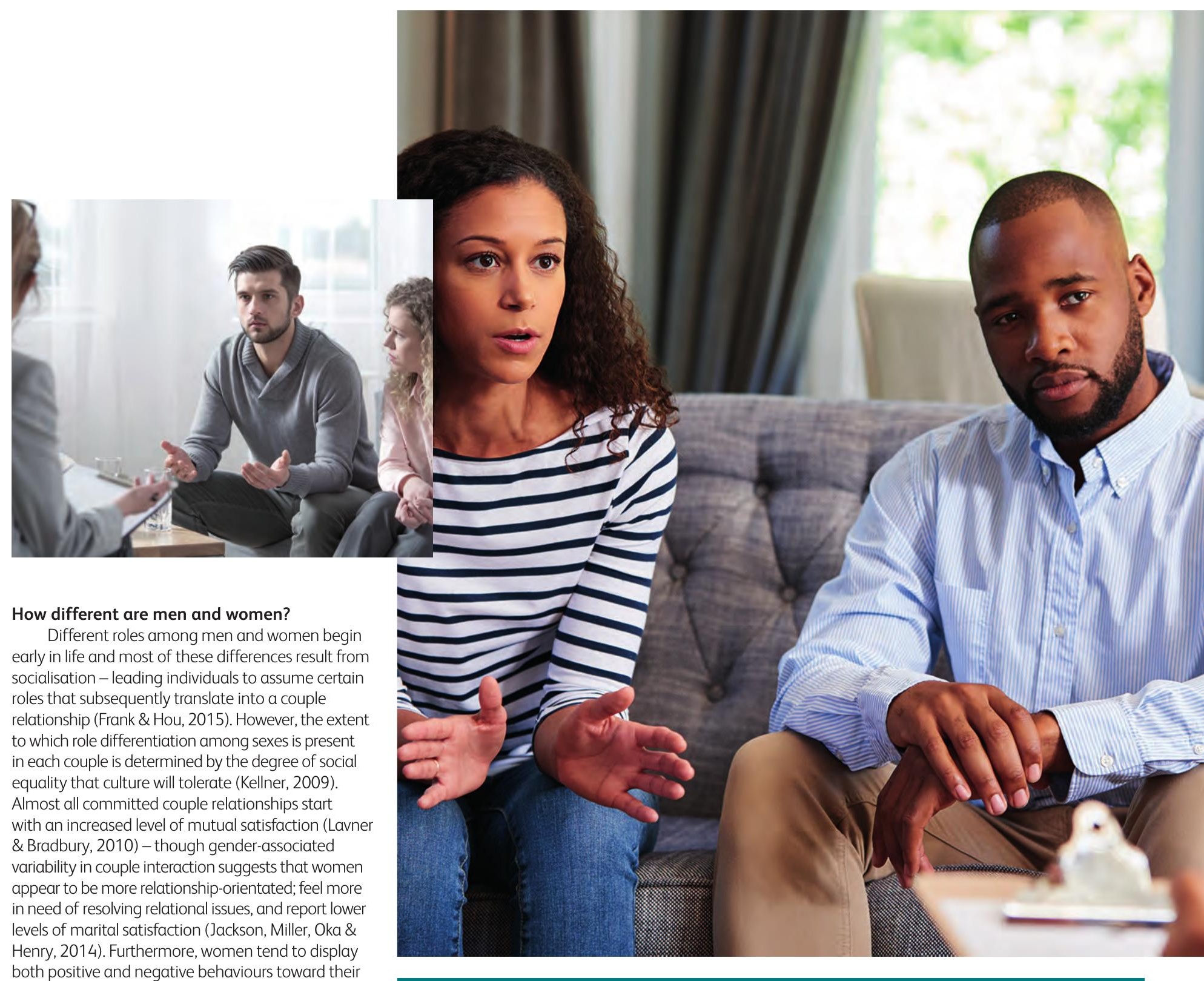

both positive and negative behaviours toward their partners, including being increasingly affirmative or expressing affect-like emotions such as sadness and anger. In contrast, males tend to show increased non-affective and instrumental behaviour such as, blame avoidance and conflict averse patterns (Gabriel, Beach \& Bodenmann, 2010).

These findings about interactions between relationship distress and individual psychological distress lead to the question: "Who is likely to make improvement from couple therapy (men or women)?" Therefore, an understanding of the impact of gender on the relationship between couples is an essential requirement for couple counsellors in delivering an efficient service. The aim of our study was to explore differences between men and women, and white and non-white clients in their levels of global psychological distress at enrolment to therapy at a Couple Relationships clinic. This report presents the findings from clients at such a clinic who were mainly residents of London or its suburbs, who had sought help due to distress in their relationships.

The psychodynamic (insight orientated)

approach is designed to assist couples explore their issues and the obstacles that interfere with change, as well as examining the reasons for their difficulties based on both conscious and unconscious contributing factors 


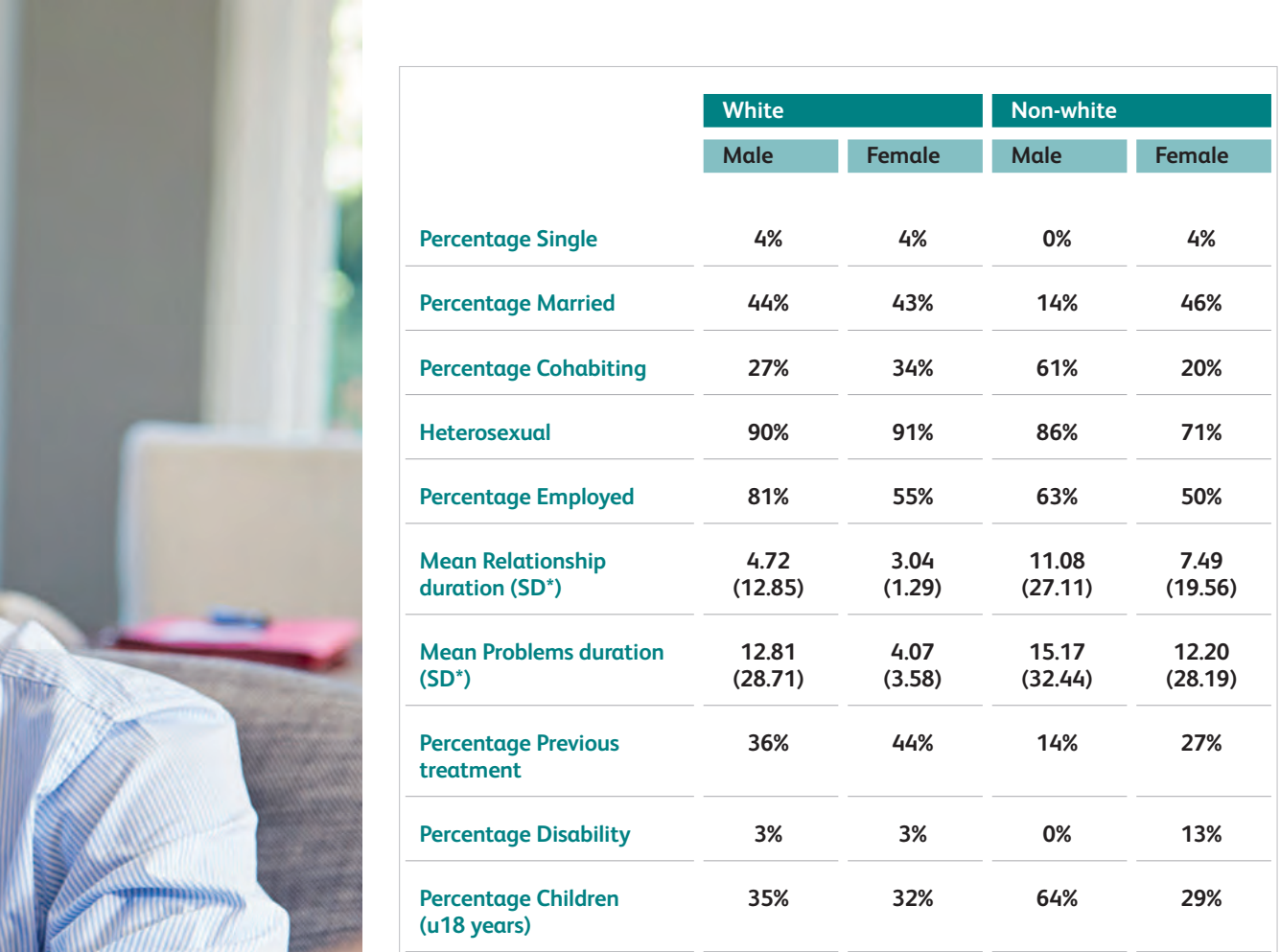

*SD - Standard Deviation

TABLE 1 Demographic and clinical characteristics of participants by ethnicity and sex for the total sample

\section{Research design and participants}

This is a retrospective cross-sectional study using the divided CORE-OM subscales (Subjective Well-being, Life Functioning, Problems/Symptoms and Risk/Harm). With the support of the team at a London Couple Relationship clinic, participants' accounts were collected between 4 January 2015 and 1 June 2017. Their ages ranged between 18 and 63 years. There were 149 women and 147 men. Most clients $(82 \%)$ identified themselves as white variants (White British/ Irish/Other White background) whilst 18\% identified as non-white (Asian/Mixed background/Black/Chinese and other ethnic backgrounds). Table 1 shows the demographic and clinical characteristics of participants by ethnicity and gender for the total sample.

\section{Measures used to collect data}

The Clinical Outcomes in Routine Evaluation - Outcome Measure (CORE-OM) is an individual mental state self-report scale, consisting of 34 questions about a participant's state of well-being over the previous week (Mellor-Clark, Barkham, Connell \& Evans, 1999). The questionnaire covers four domains: (i) Subjective Well-being ( 4 items); (ii) Problems/Symptoms (12 items); (iii) Life Functioning (12 items), and (iv) Risk/Harm (6 items). The Subjective Well-being subscale is problem orientated where higher scores indicate more distress, (although this may seem counterintuitive in relation to 'Well-being'). An example item for this domain would be 'I have felt overwhelmed by my problems'. The Problem/
Symptoms domain examines anxiety ( 4 items); depression ( 4 items); physical problems ( 2 items), and trauma ( 2 items). An example question for this dimension would be 'I have felt tense, anxious or nervous'. The Life Functioning dimension contains questions about general functioning ( 4 items); close relationships ( 4 items), and social relationships ( 4 items), for example: 'I have felt able to cope when things go wrong'. The Risk/ Harm subscale includes four items measuring 'risk to self' and two covering 'risk to others', for example: 'I have thought of hurting myself' and 'I have been physically violent to others'.

Couples attend therapy due to a range of reasons and the CORE-OM captures all these areas of difficulty. Subjective Well-being can include a general increase/decrease in functioning; a better/ worse sense of meaning in life, and improved/ impaired relationships with others (which may mean an increase/decrease in the tolerance of things that are not optimal, rather than their removal (Dirmaier, Harfst, Koch \& Schulz, 2006)). Eight items of the overall CORE-OM are positively framed, producing a mean score indicating 'Global psychological distress' - where psychological distress is measured on a 5-point Likert scale ranging from 0 ("Not at all") to 4 ("Most or all of the time"). Total scores can range from 0 to 40, where higher numbers represent more distress. Overall mean scores can be classified in the following manner to signify the distinct levels of psychological distress: 0-5 "Healthy", 6-9 "Low level", 10-14 "Mild", 15-19 “Moderate", 20-24 "Moderately severe" and 25-40 "Severe".

\section{A classic couple therapy approach}

Our data was obtained from a relationship clinic that applies a psychodynamic orientated approach (for a detailed description of this therapy refer to Hewison, Casey \& Mwamba, 2016). The psychodynamic (insight orientated) approach is designed to assist couples explore their issues and the obstacles that interfere with change, as well as examining the reasons for their difficulties based on both conscious and unconscious contributing factors (Balfour \& Lanman, 2012). With reference to this approach, the partners in a couple share a common mechanism of psychological functioning (which may be unconscious), such that a psychodynamic orientated clinician can examine the couple's interaction and make a reliable observation of the quality of their functioning as one entity (Kächele, Schachter \& Thomä, 2011). The number of couple therapy sessions varied from 0 to 44 . 


\section{Findings}

Overall, our results (see Figure 1 and Table 2) showed that women reported better Subjective Well-being compared to men. However, the levels of 'Life Functioning', 'Problems/Symptoms' or 'Risk/ Harm' failed to indicate any differences between men and women or white and non-white groups.

\section{Are there sex and ethnic differences in subjective well-being at enrolment?}

This is the first study to divide the CORE-OM questionnaire to explore perceptions of one's wellbeing, problems, day-to-day functioning and risky thoughts. Findings suggest that white individuals seek professional help for their relationship problems more often than their non-white counterparts, as reflected by the ethnic profile of clients approaching counselling services in the UK (Jones, 2014). In addition, results demonstrate that women and men differ significantly in their levels of wellbeing (as it has been shown that women enrolling to couple therapy are more distressed than their partners). Most importantly, Figure 1 demonstrates that non-white women report higher levels of distress than their white counterparts, although underrepresented in the current study. Psychological distress in men shows a similar trend. These findings reinforce the view that different family values exist and we refer to their role in explaining the degree of the impact on the non-white group's views of life and the counselling process. However, according to what we found in our study, ethnicity provides very little indication about what may be the reasons for enrolling in couple therapy, as well as what makes therapy acceptable and beneficial.

On the question of whether women or men, and respectively white or non-white individual characteristics, are the trigger for distress in couples, it is perhaps worthy to suggest that the stress process between men and women is more relevant than the ethnicity factor (Jones, 2014). However, generally speaking, it is feasible to ask; do women experience institutionalised sex discrimination in the home? Could this be one explanation to the current study findings? Or could it be that culture type (individualistic vs. collectivistic); individual characteristics (masculine vs. feminine); financial strain, or empathy between partners mediate the effect of culture on partners' sex and sex on crossover stress.

A possible explanation for these results could be that although there is an assumed availability of social support (eg. family and relatives) in the non-white women group (Taylor, Chatters, Woodward \& Brown, 2015), this could present an advantage as well as a disadvantage to them as it may deter them from approaching professional help (Williamson, 2014). This may well account for the limited number of non-white clients enrolling on couple therapy.

\begin{tabular}{|c|c|c|c|c|}
\hline Subscales & Sex/Ethnicity & Mean & SD & $\mathbf{N}$ \\
\hline Subjective & White Female & 7.28 & 3.65 & 107 \\
\hline \multirow{5}{*}{ Wellbeing } & Non-white Female & 7.63 & 3.92 & 24 \\
\hline & Total & 7.34 & 3.69 & 131 \\
\hline & White Male & 6.31 & 3.48 & 113 \\
\hline & Non-white Male & 6.18 & 3.83 & 28 \\
\hline & Total & 6.28 & 3.54 & 14 \\
\hline Life & White Female & 14.28 & 7.42 & 100 \\
\hline \multirow[t]{5}{*}{ Functioning } & Non-white Female & 17.13 & 9.30 & 24 \\
\hline & Total & 14.83 & 7.86 & 124 \\
\hline & White Male & 14.93 & 7.47 & 110 \\
\hline & Non-white Male & 15.69 & 6.87 & 26 \\
\hline & Total & 15.07 & 7.34 & 144 \\
\hline \multirow[t]{6}{*}{ Problems } & White Female & 19.44 & 10.03 & 101 \\
\hline & Non-white Female & 20.71 & 10.31 & 24 \\
\hline & Total & 19.68 & 10.05 & 125 \\
\hline & White Male & 18.38 & 9.55 & 108 \\
\hline & Non-white Male & 18.00 & 10.90 & 28 \\
\hline & Total & 18.30 & 9.81 & 136 \\
\hline \multirow[t]{6}{*}{ Risk } & White Female & .79 & 1.61 & 106 \\
\hline & Non-white Female & 1.50 & 2.67 & 24 \\
\hline & Total & .92 & 1.86 & 130 \\
\hline & White Male & 1.10 & 1.99 & 113 \\
\hline & Non-white Male & .82 & 1.22 & 28 \\
\hline & Total & 1.04 & 1.86 & 141 \\
\hline
\end{tabular}

TABLE 2 Mean and Standard Deviation for the four individual subscales across sex and ethnicity

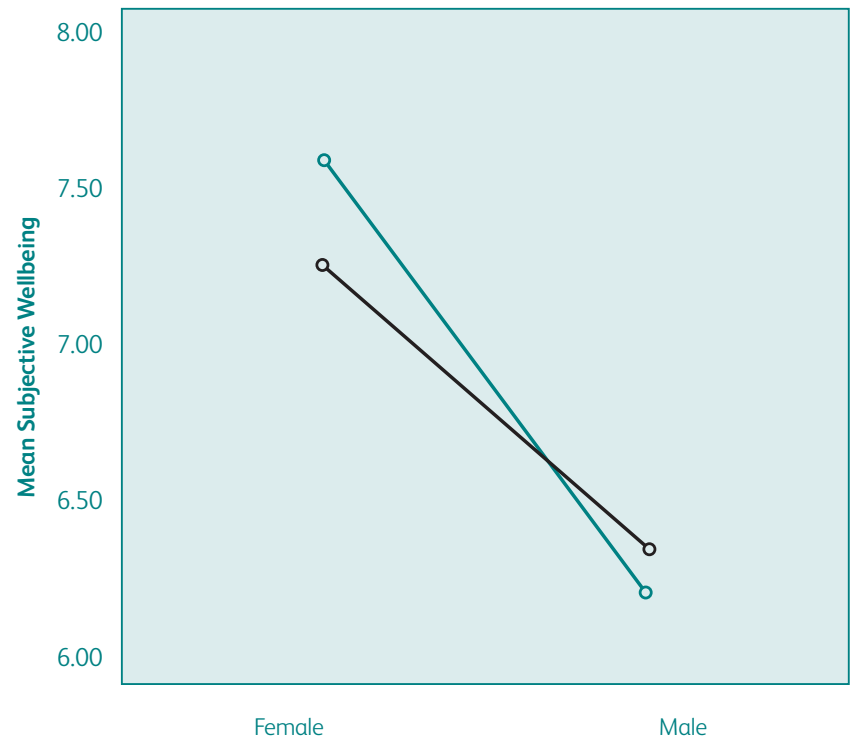

FIGURE 1 The effect of sex on Subjective Wellbeing 


\section{Communication and acceptance}

Communication sits at the basis of every relationship and couples often employ different communication patterns (such as the demandwithdraw pattern, where one partner demands and criticises, while the other becomes withdrawn and avoids confrontation (Caughlin \& Scott, 2010)). Interestingly, women are shown to be in the demanding position more often while men are happy to adopt a more withdrawn role (Holley, Sturm \& Levenson, 2010).

Psychotherapy could also provide an opportunity for couples to explore a partner's acceptance of differences. In couple therapy sessions it is important for the couple to recognise differences in their partner's views, through gaining a better understanding of how culture and gender can have an impact on one's personal value system. In this way, couples can negotiate changes by finding a middle ground without having to compromise crucial needs. Also, couple dissatisfaction has been shown to have a negative effect on physical and mental health, resulting in distressing behaviours and disrupted relationship dynamics (Robles, 2014). Therefore, if couples are supported in recognising and accepting their partner's thoughts, feelings, behaviours and values, couple therapy will prove to be an invaluable tool in modifying partners' perceptions of each other and in achieving greater acceptance of difference.

\section{Conclusion}

The naturalistic sample in this study offered an

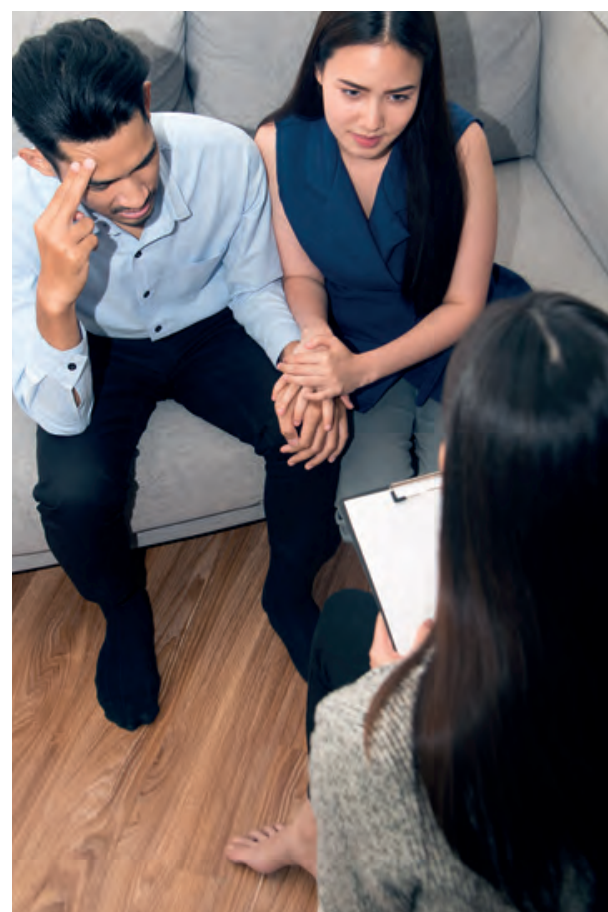
exclusive opportunity in terms of assessing the cases of existing clients, enhancing the study's external validity. The splitting of the four CORE-OM subscales, provided a unique advantage in the thorough exploration of the differences in clients' scores across gender and ethnicity. The main findings suggest that sex had an influence on subjective wellbeing at enrolment to couple therapy with women tending to be more distressed than men - particularly so for non-white women. However, the non-white fraction of clients from the total sample was very limited, therefore generalising the results across this group would not be appropriate. Nevertheless, what makes the Subjective Well-being subscale particularly relevant is that it captures the impact of a distressed relationship. Future research should therefore investigate the experience of participants using qualitative measures to capture more fully individual differences in experiencing couple distress.

\section{References}

Bacigalupe, G. \& Cámara, M. (2012) Transnational families and social technologies: Reassessing immigration psychology. Journal of Ethnic and Migration Studies, 38(9): 1425-1438 Balfour, A. \& Lanman, M. (2012) An evaluation of time-limited psychodynamic psychotherapy for couples: A pilot study. Psychology and Psychotherapy: Theory, Research and Practice, 85(3): 292-309

Caughlin, J. \& Scott, A. (2010) 'Toward a communication theory of the demand/withdraw pattern of interaction in interpersonal relationships' in Smith, S.W. \& Wilson, S.R. (Eds) New directions in interpersonal communication research, Sage: California, USA

Dirmaier, J., Harfst, T., Koch, U. \& Schulz, H. (2006) Therapy goals in inpatient psychotherapy: Differences between diagnostic groups and psychotherapeutic orientations. Clinical Psychology and Psychotherapy, 13: 34-46

Frank, K. \& Hou, F. (2015) Source-country gender roles and the division of labour within immigrant families. Journal of Marriage and Family, 77(2): 557-574

Gabriel, B., Beach, S. \& Bodenmann, G. (2010) Depression, marital satisfaction and communication in couples: Investigating gender differences. Behaviour Therapy, 41(3): 306-316

Hewison, D., Casey, P. \& Mwamba, N. (2016) The effectiveness of couple therapy: Clinical outcomes in a naturalistic United Kingdom setting. Psychotherapy, 53(4): 377-387

Holley, S., Sturm, V. \& Levenson, R. (2010) Exploring the basis for gender differences in the demand-withdraw pattern. Journal of Homosexuality, 57(5): 666-684

Jackson, J., Miller, R., Oka, M. \& Henry, R. (2014) Gender differences in marital satisfaction: A meta-analysis. Journal of Marriage and Family, 76(1): 105-129

Jones, A. (2014) Depression, race, gender and covenant marriage: An analysis of newly married couples. Health Sociology Review, 23(3): 190-207

Kächele, H., Schachter, J. \& Thomä, H. (2011) From psychoanalytic narrative to empirical single case research Implications for psychoanalytic practice (Vol. 30). Taylor \& Francis: UK

Kellner, J. (2009) Gender perspective in cross-cultura couples. Clinical Social Work Journal, 37(3): 224-229

Kenney, K. \& Kenney, M. (2014) 'Counselling multiracial individuals and families'. In Ratts, M.] \& Pedersen, P.B. (Eds) Counselling for multiculturalism and social justice: Integration, theory, and application, American Counseling Association: VA, USA

Lavner, J. \& Bradbury, T. (2010) Patterns of change in marital satisfaction over the newlywed years. Journal of Marriage and Family, 72(5): 1171-1187

Mellor-Clark, J., Barkham, M., Connell, J. \& Evans, C. (1999) Practice-based evidence and standardized evaluation: Informing the design of the CORE system. The European Journal of Psychotherapy, Counselling \& Health, 2(3): 357-374

Robles, T. (2014) Marital quality and health: Implications for marriage in the 21 st century. Current Directions in Psychological Science, 23(6): 427-432

Taylor R.] Chatters, LM. Woodward, A.T \& Brown, E. (2013) Racial and ethnic differences in extended family, friendship, fictive kin and congregational informal support networks. Family Relations, 62 (4): 609-624

Williamson, M E. (2014) The reluctance of African-Americans to engage in therapy [unpublished thesis]. Public Access Theses and Dissertations from the College of Education and Human Sciences. University of Nebraska-Lincoln

\section{About the authors}

Yana Antova, research student in Health Psychology, and Anne Manyande, Professor of Health Psychology, University of West London

\section{Keywords}

Couples, psychological distress, ethnicity, gender. 